\title{
neuroRAD 2009: the German Society of Neuroradiology Is Ready to Go
}

In early October this year, the German Society of Neuroradiology (DGNR) and guests gathered for 3 days in Cologne, Germany, for their Annual Meeting. The meeting was carefully prepared under the leadership of the congress president, Professor Michael Forsting, Chair of Radiology and Neuroradiology, University of Essen, Germany. Together with the program committee, he chose widespread diseases, like stroke and multiple sclerosis, and backache and headache, as main topics that were addressed in key lectures and scientific papers. One highlight was a lecture about curiosities in neuroradiologic findings presented by Professor Dirk Petersen, Lübeck, Germany. Each topic was prepared in advance by well-known experts who wrote articles being published on the congress website ( $w w w$. neurorad.de) and gave interviews that could be downloaded from the website. In addition, the congress provided a special program for technicians with special interest in Neuroradiology. Attracted by these activities and regular newspapers, 1,366 attendies, among them 914 physicians, 378 technicians, and 74 representatives from big companies, followed the invitation to attend the meeting in Cologne: neuroRAD 2009. (By comparison, the concomitant Annual Meeting of the British Neuroradiologists had less than 100 participants.) Among the physicians, Neurologists were the largest group of specialists $(34.9 \%)$, followed by general Radiologists (34.0\%), and Neuroradiologists $(27.4 \%)$. The rating of the meeting was between excellent and very good on the average. Being asked for their feedback, many colleagues expressed their great interest in continuing education. The DGNR has increased to 610 members and plans to keep this new meeting format and the location for the next years with the professional support of Thieme-Congress.

What can we learn from this recent experience? The topics of Neuroradiology - the paradigm of an organand diseased-oriented Radiology (Klaus Sartor) - are of high interest for all specialists in Neuromedicine. Moreover, its methods and diagnostic strategies are a must for each general Radiologist in private practice or smaller hospital that cannot afford a Neuroradiologist. Com- puted tomography (CT) and magnetic resonance imaging (MRI) of the central nervous system represent, today, more than $50 \%$ of all radiologic examinations. Common emergencies like ischemic and hemorrhagic stroke require neuroradiologic interventions. Detailed diagnosis of brain diseases with the demonstration of specific pathology and observation of pathophysiology is now possible with the means of CT, CT angiography, CT brain perfusion measurement, MRI, MR angiography, MR perfusion measurement, diffusion-weighted imaging, diffusion tensor imaging (DTI), susceptibility-weighted imaging, MR spectroscopy, and blood oxygen level-dependent MRI. The DGNR has learned that more Neuroradiologists are needed to cope with all these tasks. Neuroradiologists need a deep understanding of Neuroanatomy and Neuropathology and its molecular basis. Moreover, they need interest and understanding of the advanced methods they are applying like, e.g., functional MRI or DTI.

The DGNR, therefore, intends to further provide this platform for continuous education and presentation of good science with the help of neuroimaging. We are aware that we should share our experience with other specialists in Neuromedicine and attract Radiologist to specialize in Neuroradiology. The General Assembly invited technicians to become members of the society and offered a reduced fee. We are very glad and proud that our new concept was so much appreciated. The Annual Meetings last 2.5 days only. For the rest of the year, we wish that our colleagues in Radiology and Neuromedicine will give us support so that we can further develop structures for training, research, and patient care in our institutions. The discussions at neuroRAD 2009 have demonstrated that this is really worth doing.

\section{Rüdiger von Kummer, Dresden, Germany}

Clin Neuroradiol 2009;19:257

DOI 10.1007/s00062-009-4016-4

Published Online: November 21, 2009 